\title{
Dimensions of the Port Performance: A Review of Literature
}

\author{
Umur BUCAK ${ }^{1}$, İbrahim Müjdat BAŞARAN² ${ }^{2}$, Soner ESMER ${ }^{3,4}$
}

\author{
${ }^{1}$ Zonguldak Bulent Ecevit University, Maritime Faculty, Turkey \\ ${ }^{2}$ Zonguldak Bulent Ecevit University, Faculty of Economics and Administrative Sciences, Turkey \\ ${ }^{3}$ Iskenderun Technical University, Barbaros Hayrettin Naval Architecture and Maritime \\ Faculty, Turkey \\ ${ }^{4}$ Dokuz Eylül University, Maritime Faculty, Turkey \\ bucak.umur@beun.edu.tr; ORCID ID: https://orcid.org/0000-0001-5112-8133 \\ imbasaran@beun.edu.tr; ORCID ID: https://orcid.org/0000-0001-6608-273X \\ soner.esmer@iste.edu.tr; ORCID ID: https://orcid.org/0000-0002-0614-7818 \\ Corresponding Author: Umur BUCAK
}

\begin{abstract}
The port performance has frequently been studied in the academic literature, and the first studies on the subject are focused on financial or operational dimensions. However, today, port performance has become multi-dimensional due to the changing roles of the ports to its stakeholders, and the fact that local competition has been replaced by global competition through continuously developing routes, etc. Within this study, it is aimed to determine each dimension of the port performance concept which had been handled as a multi-dimensional process in recent years in literature. For this purpose, port performance literature is reviewed and frequency analysis of the related studies was made. As a result of the analysis, dimensional perspective was brought to the port performance concept and the indicators of each dimension used in empirical studies were gathered together. So, the concept of port performance had been divided into four basic dimensions which are operational, financial, sustainable, and logistics. Finally, dimensional gaps in port performance literature were revealed and some suggestions were given for further studies.
\end{abstract}

\section{Keywords}

Port Performance, Performance Dimensions, Performance Measurement, Operational Performance, Sustainable Performance.

\section{Introduction}

Developments such as the expansionary force of globalization, the transfer of the seat of efficient units to the countries with low input costs, the adaptation of market economies by more countries, the mounting pressure on decreasing transportation costs, the market for agility in transportation, the politic and structural changes including more autonomy in port management, the inclusion of state of the art technology in loading and discharging process, etc. require ports to be more efficient and advantageous [1].

To cite this article: Bucak, U., Başaran, I. M. \& Esmer, S. (2020). Dimensions of the Port Performance: A Review of Literature. Journal of ETA Maritime Science, 8(4), 214-240. 
These developments also increased the performance of the competition between ports [2]. While high port competition and increased carrying capacities of ships demand a better port performance, this performance largely depends on port characteristics such as infrastructure, expertise in cargo handling, shipping services, and the level of integration into freight networks [3]. In short, in today's supply chain era, both the demands of customers and the necessities of the global competitive environment force the ports to continuously improve their performance [4]. Therefore, ports need to measure their performance at regular intervals to improve their performance. In general, ports need performance measurement to measure their efficiency, effectiveness, how they have been compared to previous years, whether they have met their targets, their situation against competitors, and to gain new customers by promoting their business [5].

Ports are the hubs of the shipping, so, the performance of a port has direct and indirect effects. Therefore, the measurement and the monitoring of the ports' performance are very important to maintain the development and economic success of the countries [6]. Performance measurement results are the most important data input for regional port planning and operations [7][8]. In this age when creditworthiness is difficult, one of the most important challenges for port management is defining and prioritizing investments [9]. In response to this, regular performance measurement is one of the most important tools to meet this challenge. Thus, the investments can be easily managed according to the demands and trends of the market tracked by regularly monitored port performance.

While ports had been a shelter for ships or a facility that carried out the loading and unloading operations of the ships in the past, they have turned into a living space for all foreign trade stakeholders and a business unit that serve a large number of customers and produce valueadded businesses covering almost all logistics services. Therefore, it will be more appropriate to consider the dimensions of port performance as interdependent components, considering today's complex port management [4]. For example, while traditional measurements focus only on the seaward of the port, there is also a need to measure the connection level of the onshore [10]. Many of the studies take into account operational and financial indicators when evaluating port performance [11]. However, evaluating the port performance only in these two dimensions will not be suitable for the complex structure of the ports in terms of the services they provide to ships, cargoes, and other transportation modes [12]. Studies in recent years show that performance measurement has evolved towards focusing on a large number of indicators rather than only financial measurements and focusing on macrolevel (national) performance rather than micro-level (organization) or regional-level (industry) performance [13]. Based on this, Onwuegbuchunam [14] argued that new port performance indicators should be developed because of the changing roles of the ports. Objective criteria are required to make a meaningful performance assessment of the world's leading container ports [15]. Accordingly, UNCTAD [16] revealed that port performance has a financial, market (customer) based, human resources, and operational dimensions.

This study aims to review port performance literature and exhibit all dimensions of port performance and its indicators. For this purpose, the whole reached articles that measured ports' performance or reviewed related measurement tools were researched thoroughly. 
Accordingly, in the second section, the methodology of this study and review process were presented, and the review of the literature made in the scope of 'performance measurement', 'internal and external factors that affect port performance', 'milestones in port performance measurement' and results obtained from the detailed review was expressed. In the third section, each indicator of each performance dimension used in the port performance literature was detected. Finally, similar studies taken part in related literature were evaluated and the results of the research were discussed.

\section{Methodology}

In this study, port performance-related literature was presented by reviewing academic articles issued in academic journals which are available at the 'Google Scholar'. 'Google Scholar' database was selected for review because no different studies were found in other academic databases. So, the search was made by combining the words 'port' and 'performance' in the 'Google Scholar' database considering articles after the year 2000. However, an exemption was made to Tongzon [17] and Martinez-Budria et al. [18], because they were approached as basic articles in terms of its contents. After reading abstract sections of the studies, 124 articles were seemed to be relevant for our research. A frequency analysis method was employed to examine relevant literature. First, a literature table that contains the methods of the accessed articles and the performance dimensions they assessed were revealed, and thus, the articles were classified. Then, homogeneous information obtained after the classification of the articles by dimensions was brought together. In the light of such information, dimensions of the port performance and its indicators were revealed. Besides statistical data related to the contents of the studies were analysed with the help of Microsoft Excel.

\subsection{Literature Review}

Bichou [19] classified the methods used in port performance assessment into three groups: performance measurements and index methods, economic impact studies, and efficient frontier approaches. Traditionally, port and terminal performance have been assessed by way of calculation of whether optimizing the efficiency of handling operations at the berths and terminal areas [20][21]. However, port performance can also be evaluated via calculation of its technical effectiveness or cost-effectiveness, or comparison of the port's actual output with the targeted output [22]. Herein, the measurement of the desired or expected performance dimension is critical because port performance measurement is an important tool in terms of managing relations with stakeholders and achieving a sustainable competitive position [23]. A performance measurement or metric, however, is presented numerically to quantify one or more attributes of an object, product, process, or any related factor, and should allow comparison and evaluation in contrast with objectives, criteria, and/or historical data [19].

Until the 1980s, performance measurement was mostly limited to financial measurements. Performance measurement techniques emerged through the use of a double-entry accounting system [13]. Over time, operational performance dimensions such as effectiveness, productivity, utilization, and effectiveness, which will enable measurement on an operational scale, have been added to these techniques [24]. However, today, performance measurement techniques are more complex considering the factors such as the more complex business environment, ever-changing global customer behaviour, and developing company structures. In the literature, there are two types of port performance measurement approaches, which are descriptive and empirical. Descriptive approaches provide 
information to be used to observe long-term data behaviour. On the other hand, empirical models that measure port performance are used to obtain time-series graphs, horizontal section graphs, scatter diagrams to reveal the relationships between two or more variables and the relationships between its trends [25]. At this point, Somensi et al. [26] revealed that Data Envelopment Analysis (DEA) and MultiCriteria Decision Making (MCDM) methods are frequently employed in port performance measurement studies. In addition to this, from the port selection perspective, there are two basic approaches to the evaluation of port performance. The traditional approach is based on direct measurements involving observations, interviews, surveys, while the behavioural approach focuses on the port users' decisions and measures [27].

However, due to the unique nature of the ports which are highly affected by local dynamics, an internationally accepted standard port performance measurement tool has not been developed yet. Although at the macro level, such performance measurement tools have been developed for the logistics industry. For example, the logistics performance index which is an interchangeable comparison tool, generated to help countries identify the challenges and opportunities in trade logistics, is a measurement tool developed by the World Bank and recognized in the international logistics world [28]. On the other hand, the project called 'PPRISM' put forward by the European Commission is the most concrete study that tried to set the port performance measurement to a standard. After all, this project cannot fully meet the needs due to its problems in terms of digitising performance dimensions [78].

Although port performance is one of the most popular topics in the literature, there is no consensus on which factors affect port performance. While some researchers think that administrative factors have an impact on port performance, some researchers relate between the port performance and management structure, geographic factors, the port's socio-economic environment, or the local supply chain system [29]. Studies thatpointed out the importance of thelocation [30][31][32] emphasized that the ports in different regions perform differently. One of the most important elements in the external environment of the port is the political environment. Some studies [33][34][35] suggested that political decisions determine port performance to some extent. Some studies [31][36] defended that ports should obtain economies of scale by increasing the capacity to improve their performance. At this point, it would not be correct to confine the capacity concept to physical capacity. While expressing the linear relationship of the capacity with port performance, some authors [37][38][39] brought the economic capacity of the port environment into the forefront, some of them [6][40] emphasized information and technology capacity, and one of them [41] pointed out the port's service capacity. Accordingly, many authors think that the factors that determine the quality of the port infrastructure and superstructure, such as length, design, and maintenance of the infrastructure and superstructure, availability, seaside accessibility, etc. affect the performance [33][42][43][44]. On the contrary, Pak et al. [45] advocated the exact opposite and stated that the intangible resources such as recognition, technology knowledge, effective process, and qualified personnel fundamentally affect the port performance.

Performance perceptions of ports have changed as well as the evolution of ports over the years. In this sense, there are milestone articles in the literature thanks to their contributions to the concept of port performance. Tongzon [17] was the first to reveal the determinants of the port performance. Bichou and Gray [10] discussed that exclusively financial and production-based evaluations on port 
performance remain incapable to determine customer satisfaction levels. Cullinane et al. [7] had one of the unique studies that processed performance inputs and outputs long term and evaluated with panel data analysis. Darbra et al. [53] were first-timers to inject sustainability concerns in the port performance concept. Woo et al. [68] expressed that port performance is versatile, cannot be limited to internal processes, and is linked to external service aspects such as service quality and logistics aspects. Madeira et al. [71] presented the first known study that employed one of the MCDM methods to evaluate the performance of ports. De Langen and Sharypova [78] became the initial researchers who used the 'intermodal linklevel' as one of the performance indicators. $\mathrm{Li}$ and Jiang [91] presented the first known study that handled the collaboration performance of the ports with its dry ports. Antao et al. [100] approached safety performance as a separate port performance item. Musso and Sciomachen [121] proposed solutions for alleviating mega vessels' effects on port performance.

Today's ports operate as logistics centres and even trade centres as a result of the increasing volume of cargo transported with the spread of trade to all countries. This situation brings competition among the ports in its wake. On the one hand, Cullinane and Wang [46] believed that inter-port competition will encourage ports to improve its performance within the framework of the Orthodoxeconomic theory. On the other hand, Cheon et al. [47] argued that competition increases performance at first, but over time this pressure will exceed a certain threshold and will downgrade performance. As a result of competitiveness pressures such as the increase in ship sizes and the variety of cargoes that can be containerized in recent years, dry ports have been used in container terminals' hinterland. Dry ports, with its additional areas and facilities, shorten waiting times at the port, regulate cargo traffic, provide container segregation and transportation options, so increase the capacity of the port, approximate the ports to its hinterland, ensure that the ports offer services diversity, and enhance the foreign trade capacity of the region by bringing the ports closer to the manufacturer [48]. For this reason, it is expected that dry ports have a positive impact on port performance by increasing their efficiency, the number of ship calls, reliability, and berth productivity. As another way of dealing with this competitive pressure, Han [49] proposed that ports should cooperate with supply chain partners to provide value-added services to their customers. However, ports should cooperate with not only supply chain service providers. Within the port area, customers (consignors, consignees), regulatory groups (freight forwarders, logistics service providers), physical groups (terminal operators), authoriser groups, and financial groups (insurance companies) need to interact with each other horizontally and vertically [50]. In this sense, the management of these relations can directly affect port performance. For this reason, Hervas-Peralta et al. [51] who pointed out the right planning stated that port performance will be increased if the focus is on terminal area optimization. In support of this, Esmer [5] highlighted the internal factors such as handled empty containers, inefficient container movements (displacement movements within the bay), the automation level of the ship to shore cranes, container weight, and the necessities for special requirements as well as commercial constraints.

\subsection{Results}

As a result of the frequency analysis, information such as the year and the journal in which the articles were published, the methods in which the articles were employed, and the performance dimensions in which the articles revealed while measuring the port performance were classified and shown in Table 1. 
Table 1. Literature Table

\begin{tabular}{|c|c|c|c|c|}
\hline Year & Reference & Journal & Method(s) & $\begin{array}{c}\text { Approached Performance } \\
\text { Dimension }\end{array}$ \\
\hline 1995 & [17] & $\begin{array}{c}\text { Transportation Research } \\
\text { Part A }\end{array}$ & Mathematical model & $\begin{array}{l}\text { infrastructure and } \\
\text { superstructure, operation, } \\
\text { financial }\end{array}$ \\
\hline 1999 & [18] & $\begin{array}{l}\text { International Journal of } \\
\text { Transport Economics }\end{array}$ & DEA & financial, operation \\
\hline 2001 & {$[33]$} & $\begin{array}{c}\text { Transportation Research } \\
\text { Part A }\end{array}$ & DEA & $\begin{array}{l}\text { operation, financial, } \\
\text { infrastructure and } \\
\text { superstructure }\end{array}$ \\
\hline 2002 & {$[42]$} & $\begin{array}{c}\text { Review of Urban \& } \\
\text { Regional Development } \\
\text { Studies }\end{array}$ & DEA & $\begin{array}{c}\text { operation, infrastructure and } \\
\text { superstructure }\end{array}$ \\
\hline 2004 & {$[10]$} & $\begin{array}{c}\text { Maritime Policy \& } \\
\text { Management }\end{array}$ & Structured Interview & $\begin{array}{c}\text { operation, financial, customer } \\
\text { satisfaction }\end{array}$ \\
\hline 2004 & {$[41]$} & $\begin{array}{c}\text { Maritime Economics \& } \\
\text { Logistics }\end{array}$ & DEA & $\begin{array}{l}\text { operation, infrastructure and } \\
\text { superstructure, financial, } \\
\text { customer satisfaction }\end{array}$ \\
\hline 2004 & [7] & $\begin{array}{l}\text { The Review of Network } \\
\text { Economics }\end{array}$ & $\begin{array}{l}\text { DEA and Panel data } \\
\text { analysis }\end{array}$ & $\begin{array}{l}\text { operation, infrastructure and } \\
\text { superstructure }\end{array}$ \\
\hline 2004 & {$[52]$} & $\begin{array}{c}\text { Journal of Marine Science } \\
\text { and Technology }\end{array}$ & $\begin{array}{l}\text { Hierarchic score } \\
\text { method, Grey } \\
\text { relational analysis, }\end{array}$ & $\begin{array}{l}\text { operation, financial, } \\
\text { infrastructure and } \\
\text { superstructure }\end{array}$ \\
\hline 2004 & {$[53]$} & Marine Pollution Bulletin & Literature review & sustainability \\
\hline 2005 & {$[54]$} & $\begin{array}{c}\text { Transportation Research } \\
\text { Part A }\end{array}$ & $\begin{array}{l}\text { stochastic frontier } \\
\text { analysis }\end{array}$ & $\begin{array}{c}\text { operation, financial, customer } \\
\text { satisfaction, infrastructure and } \\
\text { superstructure, }\end{array}$ \\
\hline 2006 & {$[55]$} & $\begin{array}{l}\text { International Journal of } \\
\text { Logistics: Research and } \\
\text { Applications }\end{array}$ & DEA & $\begin{array}{l}\text { operation, infrastructure and } \\
\text { superstructure }\end{array}$ \\
\hline 2006 & {$[20]$} & Transportation Research & $\begin{array}{l}\text { stochastic frontier } \\
\text { analysis and DEA }\end{array}$ & $\begin{array}{c}\text { infrastructure and } \\
\text { superstructure, operation }\end{array}$ \\
\hline 2006 & {$[22]$} & $\begin{array}{c}\text { Research in } \\
\text { Transportation } \\
\text { Economics }\end{array}$ & Literature review & financial, operation, safety \\
\hline 2006 & [19] & $\begin{array}{l}\text { Research in } \\
\text { Transportation } \\
\text { Economics } \\
\end{array}$ & Literature review & $\begin{array}{c}\text { financial, operation, customer } \\
\text { satisfaction }\end{array}$ \\
\hline 2007 & {$[56]$} & $\begin{array}{l}\text { Applied Mathematics and } \\
\text { Computation }\end{array}$ & Fuzzy MCDM & $\begin{array}{l}\text { infrastructure and } \\
\text { superstructure, operation, } \\
\text { financial }\end{array}$ \\
\hline 2007 & {$[57]$} & $\begin{array}{c}\text { Research in } \\
\text { Transportation } \\
\text { Economics }\end{array}$ & Literature review & $\begin{array}{l}\text { operation, infrastructure and } \\
\text { superstructure, financial, } \\
\text { customer satisfaction, safety }\end{array}$ \\
\hline 2007 & [15] & $\begin{array}{l}\text { Maritime Policy \& } \\
\text { Management }\end{array}$ & DEA & operation \\
\hline
\end{tabular}


Table 1. Literature Table (Cont')

\begin{tabular}{|c|c|c|c|c|}
\hline Year & Reference & Journal & Method(s) & $\begin{array}{c}\text { Approached Performance } \\
\text { Dimension }\end{array}$ \\
\hline 2008 & [58] & $\begin{array}{l}\text { Maritime Policy \& } \\
\text { Management }\end{array}$ & Literature review & $\begin{array}{l}\text { operation, financial, } \\
\text { infrastructure and } \\
\text { superstructure }\end{array}$ \\
\hline 2008 & [59] & $\begin{array}{c}\text { Maritime Policy \& } \\
\text { Management }\end{array}$ & Mathematical model & operation \\
\hline 2008 & {$[43]$} & $\begin{array}{l}\text { European Journal of } \\
\text { Scientific Research }\end{array}$ & $\begin{array}{c}\text { DEA, Correlation } \\
\text { Analysis, Regression } \\
\text { Analysis }\end{array}$ & $\begin{array}{c}\text { operation, infrastructure and } \\
\text { superstructure }\end{array}$ \\
\hline 2008 & {$[60]$} & $\begin{array}{c}\text { Transportation Research } \\
\text { Part A }\end{array}$ & Factor Analysis & $\begin{array}{l}\text { operation, financial, } \\
\text { infrastructure and } \\
\text { superstructure, customer } \\
\text { satisfaction, logistics }\end{array}$ \\
\hline 2008 & [5] & $\begin{array}{c}\text { Dokuz Eylül Üniversitesi } \\
\text { Sosyal Bilimler Enstitüsü } \\
\text { Dergisi }\end{array}$ & Literature review & $\begin{array}{c}\text { operation, infrastructure and } \\
\text { superstructure, financial }\end{array}$ \\
\hline 2009 & [8] & $\begin{array}{l}\text { IUP Journal of } \\
\text { Infrastructure }\end{array}$ & $\begin{array}{c}\text { Correlation Analysis, } \\
\text { Principal Component } \\
\text { Analysis }\end{array}$ & $\begin{array}{c}\text { operation, infrastructure and } \\
\text { superstructure }\end{array}$ \\
\hline 2009 & [61] & $\begin{array}{l}\text { Journal of Cleaner } \\
\text { Production }\end{array}$ & Literature review & sustainability \\
\hline 2009 & {$[32]$} & $\begin{array}{l}\text { Maritime Policy \& } \\
\text { Management }\end{array}$ & DEA & $\begin{array}{c}\text { operation, infrastructure and } \\
\text { superstructure }\end{array}$ \\
\hline 2010 & [34] & $\begin{array}{c}\text { Journal of Economic } \\
\text { Studies }\end{array}$ & $\begin{array}{l}\text { DEA, Panel data } \\
\text { analysis }\end{array}$ & $\begin{array}{c}\text { infrastructure and } \\
\text { superstructure, operation }\end{array}$ \\
\hline 2010 & {$[62]$} & $\begin{array}{l}\text { Maritime Economics \& } \\
\text { Logistics }\end{array}$ & DEA & $\begin{array}{c}\text { infrastructure and } \\
\text { superstructure, operation }\end{array}$ \\
\hline 2010 & [63] & $\begin{array}{c}\text { International Journal } \\
\text { of Computational } \\
\text { Intelligence Systems }\end{array}$ & DEA & operation \\
\hline 2010 & {$[64]$} & $\begin{array}{c}\text { Transportation Planning } \\
\text { and Technology }\end{array}$ & $\begin{array}{l}\text { Free Disposal Hull, } \\
\text { DEA }\end{array}$ & financial \\
\hline 2010 & {$[46]$} & $\begin{array}{l}\text { Operations Research } \\
\text { Spectrum }\end{array}$ & DEA, ANOVA & $\begin{array}{c}\text { operation, infrastructure and } \\
\text { superstructure }\end{array}$ \\
\hline 2011 & [65] & $\begin{array}{l}\text { Analele Universitatii } \\
\text { "Eftimie Murgu" Resita } \\
\text { Fascicola de Inginerie }\end{array}$ & Literature review & operation \\
\hline 2011 & [66] & $\begin{array}{c}\text { Scientific Research and } \\
\text { Essays }\end{array}$ & Fuzzy MCDM & $\begin{array}{l}\text { infrastructure and } \\
\text { superstructure, operation, } \\
\text { financial }\end{array}$ \\
\hline 2011 & [67] & $\begin{array}{c}\text { Resources, Conservation } \\
\text { and Recycling }\end{array}$ & $\begin{array}{c}\text { Mathematical model, } \\
\text { DEA }\end{array}$ & $\begin{array}{l}\text { operation, financial, } \\
\text { sustainability }\end{array}$ \\
\hline 2011 & [68] & $\begin{array}{c}\text { Maritime Economics \& } \\
\text { Logistics }\end{array}$ & $\begin{array}{c}\text { Confirmatory Factor } \\
\text { Analysis }\end{array}$ & $\begin{array}{l}\text { operation, safety, customer } \\
\text { satisfaction, logistics, financial }\end{array}$ \\
\hline 2011 & [69] & Transport Policy & Fuzzy ANP & $\begin{array}{l}\text { operation, financial, } \\
\text { infrastructure and } \\
\text { superstructure }\end{array}$ \\
\hline
\end{tabular}


Table 1. Literature Table (Cont')

\begin{tabular}{|c|c|c|c|c|}
\hline Year & Reference & Journal & Method(s) & $\begin{array}{c}\text { Approached Performance } \\
\text { Dimension }\end{array}$ \\
\hline 2012 & {$[30]$} & $\begin{array}{l}\text { International Journal of } \\
\text { Physical Distribution and } \\
\text { Logistics Management }\end{array}$ & T Test & $\begin{array}{l}\text { operation, logistics, financial, } \\
\text { safety }\end{array}$ \\
\hline 2012 & {$[70]$} & $\begin{array}{l}\text { Journal of Management \& } \\
\text { Economics }\end{array}$ & Depth interview, t Test & safety, operation \\
\hline 2012 & {$[71]$} & $\begin{array}{l}\text { International Journal of } \\
\text { Production Economics }\end{array}$ & $\begin{array}{l}\text { Factor analysis, } \\
\text { MACBETH }\end{array}$ & financial, operation \\
\hline 2012 & {$[72]$} & $\begin{array}{l}\text { Simulation Modelling } \\
\text { Practice and Theory }\end{array}$ & Simulation Model & $\begin{array}{l}\text { operation, infrastructure and } \\
\text { superstructure }\end{array}$ \\
\hline 2012 & [73] & $\begin{array}{l}\text { The Asian Journal of } \\
\text { Shipping and Logistics }\end{array}$ & $\begin{array}{l}\text { Factor analysis, Fuzzy } \\
\text { logic }\end{array}$ & sustainability \\
\hline 2012 & {$[74]$} & $\begin{array}{c}\text { International Journal of } \\
\text { Business Performance } \\
\text { Management }\end{array}$ & DEA & $\begin{array}{l}\text { operation, financial, } \\
\text { infrastructure and } \\
\text { superstructure, customer } \\
\text { satisfaction, logistics }\end{array}$ \\
\hline 2012 & {$[75]$} & Transport Policy & Literature review & operation \\
\hline 2013 & {$[76]$} & $\begin{array}{l}\text { International Journal of } \\
\text { Physical Distribution and } \\
\text { Logistics Management }\end{array}$ & AHP and Fuzzy MCDM & sustainability \\
\hline 2013 & {$[77]$} & $\begin{array}{c}\text { Research in } \\
\text { Transportation Business } \\
\text { and Management }\end{array}$ & Mathematical model & sustainability, financial \\
\hline 2013 & [78] & $\begin{array}{c}\text { Research in } \\
\text { Transportation Business } \\
\text { and Management }\end{array}$ & Mathematical model & $\begin{array}{l}\text { logistics, operation, } \\
\text { sustainability, financial, } \\
\text { infrastructure and } \\
\text { superstructure }\end{array}$ \\
\hline 2013 & [79] & $\begin{array}{c}\text { Research in } \\
\text { Transportation Business } \\
\text { and Management }\end{array}$ & Correlation Analysis & operation, safety, logistics \\
\hline 2013 & {$[35]$} & $\begin{array}{c}\text { Research in } \\
\text { Transportation Business } \\
\text { and Management }\end{array}$ & Mathematical model & $\begin{array}{c}\text { infrastructure and } \\
\text { superstructure, operation }\end{array}$ \\
\hline 2013 & {$[80]$} & $\begin{array}{c}\text { Research in } \\
\text { Transportation Business } \\
\text { and Management }\end{array}$ & $\begin{array}{l}\text { Stochastic frontier } \\
\text { analysis, DEA }\end{array}$ & $\begin{array}{l}\text { operation, infrastructure and } \\
\text { superstructure }\end{array}$ \\
\hline 2013 & [81] & $\begin{array}{c}\text { Girişimcilik ve Kalkınma } \\
\text { Dergisi }\end{array}$ & Descriptive analysis & financial, customer satisfaction \\
\hline 2013 & {$[82]$} & $\begin{array}{c}\text { Supply Chain } \\
\text { Management: An } \\
\text { International Journal }\end{array}$ & $\begin{array}{l}\text { Structural equation } \\
\text { model }\end{array}$ & $\begin{array}{l}\text { operation, financial, customer } \\
\text { satisfaction, logistics }\end{array}$ \\
\hline 2013 & [83] & $\begin{array}{l}\text { Maritime Policy \& } \\
\text { Management }\end{array}$ & DEA & $\begin{array}{l}\text { sustainability, financial, } \\
\text { infrastructure and } \\
\text { superstructure, operation }\end{array}$ \\
\hline 2013 & {$[21]$} & Transport Policy & DEA & $\begin{array}{l}\text { operation, infrastructure and } \\
\text { superstructure }\end{array}$ \\
\hline 2013 & {$[84]$} & Polish Maritime Research & Interview & financial, operation, logistics \\
\hline
\end{tabular}


Table 1. Literature Table (Cont')

\begin{tabular}{|c|c|c|c|c|}
\hline Year & Reference & Journal & Method(s) & $\begin{array}{c}\text { Approached Performance } \\
\text { Dimension }\end{array}$ \\
\hline 2013 & [85] & $\begin{array}{c}\text { Maritime Economics \& } \\
\text { Logistics }\end{array}$ & DEA & $\begin{array}{c}\text { infrastructure and } \\
\text { superstructure, operation }\end{array}$ \\
\hline 2014 & [12] & Verimlilik Dergisi & DEA & $\begin{array}{c}\text { infrastructure and } \\
\text { superstructure, operation }\end{array}$ \\
\hline 2014 & {$[86]$} & Transport Reviews & Literature review & operation \\
\hline 2014 & [87] & $\begin{array}{c}\text { İstanbul Üniversitesi } \\
\text { İşletme Fakültesi Dergisi }\end{array}$ & DEA & financial, operation \\
\hline 2014 & [88] & $\begin{array}{c}\text { Transportation Research } \\
\text { Part E }\end{array}$ & Mathematical model & $\begin{array}{l}\text { operation, financial, logistics, } \\
\text { safety }\end{array}$ \\
\hline 2014 & {$[3]$} & $\begin{array}{l}\text { Maritime Policy and } \\
\text { Management }\end{array}$ & Factor analysis & operation, financial \\
\hline 2014 & [89] & $\begin{array}{l}\text { Decision Support } \\
\text { Systems }\end{array}$ & Mathematical model & operation, financial \\
\hline 2014 & {$[90]$} & $\begin{array}{c}\text { Transportation Research } \\
\text { Part A }\end{array}$ & $\begin{array}{l}\text { Hierarchical cluster } \\
\text { analysis }\end{array}$ & $\begin{array}{l}\text { operation, infrastructure and } \\
\text { superstructure, financial }\end{array}$ \\
\hline 2014 & {$[91]$} & $\begin{array}{l}\text { International Journal } \\
\text { of e-Navigation and } \\
\text { Maritime Economy }\end{array}$ & $\begin{array}{l}\text { Grey Relations } \\
\text { Analysis, AHP }\end{array}$ & $\begin{array}{c}\text { customer satisfaction, financial, } \\
\text { operation }\end{array}$ \\
\hline 2014 & [92] & Marine Pollution Bulletin & Delphi & sustainability, operation \\
\hline 2014 & [9] & $\begin{array}{l}\text { Maritime Policy \& } \\
\text { Management }\end{array}$ & $\begin{array}{c}\text { Importance - } \\
\text { Performance Analysis }\end{array}$ & $\begin{array}{l}\text { operation, safety, financial, } \\
\text { customer satisfaction }\end{array}$ \\
\hline 2014 & {$[93]$} & $\begin{array}{l}\text { International Journal } \\
\text { of Research in Applied, } \\
\text { Natural and Social } \\
\text { Sciences }\end{array}$ & Literature review & $\begin{array}{c}\text { infrastructure and } \\
\text { superstructure, logistics, } \\
\text { operation, financial }\end{array}$ \\
\hline 2015 & {$[94]$} & $\begin{array}{c}\text { Transportation Research } \\
\text { Part C }\end{array}$ & Simulation Model & $\begin{array}{c}\text { infrastructure and } \\
\text { superstructure, operation }\end{array}$ \\
\hline 2015 & {$[95]$} & $\begin{array}{l}\text { Transportation Research } \\
\text { Procedia } \\
\end{array}$ & $\begin{array}{c}\text { Multiple Regression } \\
\text { Analysis }\end{array}$ & operation \\
\hline 2015 & {$[96]$} & Alphanumeric Journal & DEA & $\begin{array}{c}\text { infrastructure and } \\
\text { superstructure, operation, } \\
\text { financial }\end{array}$ \\
\hline 2015 & {$[45]$} & $\begin{array}{l}\text { The Asian Journal of } \\
\text { Shipping and Logistics }\end{array}$ & Fuzzy TOPSIS & $\begin{array}{c}\text { operation, safety, customer } \\
\text { satisfaction }\end{array}$ \\
\hline 2015 & {$[13]$} & $\begin{array}{c}\text { International Journal of } \\
\text { Logistics Research and } \\
\text { Applications }\end{array}$ & Literature review & $\begin{array}{l}\text { operation, financial, customer } \\
\text { satisfaction, sustainability }\end{array}$ \\
\hline 2015 & {$[1]$} & $\begin{array}{l}\text { International Journal } \\
\text { of Productivity } \\
\text { and Performance } \\
\text { Management }\end{array}$ & Literature review & $\begin{array}{l}\text { operation, sustainability, } \\
\text { financial, customer satisfaction }\end{array}$ \\
\hline 2015 & [97] & Transportation Research & DEA & $\begin{array}{c}\text { infrastructure and } \\
\text { superstructure, operation }\end{array}$ \\
\hline
\end{tabular}


Table 1. Literature Table (Cont')

\begin{tabular}{|c|c|c|c|c|}
\hline Year & Reference & Journal & Method(s) & $\begin{array}{c}\text { Approached Performance } \\
\text { Dimension }\end{array}$ \\
\hline 2015 & {$[98]$} & $\begin{array}{c}\text { International Journal of } \\
\text { Operations and Logistics } \\
\text { Management }\end{array}$ & ELECTRE & $\begin{array}{l}\text { operation, financial, } \\
\text { infrastructure and } \\
\text { superstructure }\end{array}$ \\
\hline 2016 & [2] & $\begin{array}{l}\text { Benchmarking: An } \\
\text { International Journal }\end{array}$ & DEA & operation, financial \\
\hline 2016 & [99] & $\begin{array}{c}\text { IEEE Transactions } \\
\text { on Intelligent } \\
\text { Transportation Systems }\end{array}$ & Mathematical Model & operation \\
\hline 2016 & {$[100]$} & Safety Science & Literature review & safety, sustainability \\
\hline 2016 & [101] & $\begin{array}{c}\text { International Journal of } \\
\text { Logistics Research and } \\
\text { Applications }\end{array}$ & $\begin{array}{l}\text { Structural equation } \\
\text { model }\end{array}$ & logistics, operation, financial \\
\hline 2016 & {$[24]$} & $\begin{array}{c}\text { Transportation Research } \\
\text { Part A }\end{array}$ & $\begin{array}{l}\text { Stochastic frontier } \\
\text { analysis, DEA }\end{array}$ & $\begin{array}{c}\text { infrastructure and } \\
\text { superstructure, operation }\end{array}$ \\
\hline 2016 & [37] & $\begin{array}{l}\text { Maritime Policy \& } \\
\text { Management }\end{array}$ & $\begin{array}{c}\text { Factor analysis, } \\
\text { Structural equation } \\
\text { model, }\end{array}$ & sustainability, financial \\
\hline 2017 & {$[26]$} & Intangible Capital & $\begin{array}{c}\text { Literature review, } \\
\text { Bibliographical } \\
\text { portfolio analysis }\end{array}$ & logistics, operation, financial \\
\hline 2017 & [102] & $\begin{array}{c}\text { Maritime Policy and } \\
\text { Management }\end{array}$ & Delphi analysis & sustainability \\
\hline 2017 & {$[103]$} & $\begin{array}{l}\text { The Asian Journal of } \\
\text { Shipping and Logistics }\end{array}$ & AHP and Fuzzy TOPSIS & $\begin{array}{c}\text { operation, customer } \\
\text { satisfaction }\end{array}$ \\
\hline 2017 & {$[4]$} & $\begin{array}{c}\text { Transportation Research } \\
\text { Part A }\end{array}$ & AHP, DEMATEL, ANP & $\begin{array}{c}\text { operation, financial, customer } \\
\text { satisfaction, logistics, } \\
\text { sustainability }\end{array}$ \\
\hline 2017 & [104] & $\begin{array}{l}\text { Journal of Management, } \\
\text { Marketing and Logistics }\end{array}$ & DEA & $\begin{array}{l}\text { operation, infrastructure and } \\
\text { superstructure }\end{array}$ \\
\hline 2017 & {$[11]$} & $\begin{array}{l}\text { Economics and Finance } \\
\text { in Indonesia }\end{array}$ & $\begin{array}{l}\text { Hybrid Least square } \\
\text { methods }\end{array}$ & operation, financial \\
\hline 2017 & {$[105]$} & $\begin{array}{l}\text { Maritime Economics and } \\
\text { Logistics }\end{array}$ & $\begin{array}{l}\text { Mathematical model } \\
\text { and DEA }\end{array}$ & $\begin{array}{c}\text { operation, safety, infrastructure } \\
\text { and superstructure }\end{array}$ \\
\hline 2017 & [38] & $\begin{array}{c}\text { Forum Scientiae } \\
\text { Oeconomia }\end{array}$ & Literature review & financial, operation \\
\hline 2017 & {$[31]$} & $\begin{array}{c}\text { Maritime Economics and } \\
\text { Logistics }\end{array}$ & $\begin{array}{l}\text { Network analysis and } \\
\text { Panel Regression }\end{array}$ & $\begin{array}{c}\text { infrastructure and } \\
\text { superstructure, operation }\end{array}$ \\
\hline 2017 & {$[25]$} & Computer Science & $\begin{array}{c}\text { Port Efficiency } \\
\text { Performance (PEP) } \\
\text { Model }\end{array}$ & operation \\
\hline 2017 & {$[106]$} & $\begin{array}{c}\text { International Colloquium } \\
\text { on Logistics and Supply } \\
\text { Chain Management }\end{array}$ & $\begin{array}{l}\text { Principal component } \\
\text { analysis }\end{array}$ & $\begin{array}{l}\text { financial, operation, } \\
\text { infrastructure and } \\
\text { superstructure }\end{array}$ \\
\hline 2017 & [107] & $\begin{array}{l}\text { MATEC Web of } \\
\text { Conferences }\end{array}$ & $\begin{array}{l}\text { Stochastic Simulation } \\
\text { Model }\end{array}$ & operation \\
\hline
\end{tabular}


Table 1. Literature Table (Cont')

\begin{tabular}{|c|c|c|c|c|}
\hline Year & Reference & Journal & Method(s) & $\begin{array}{c}\text { Approached Performance } \\
\text { Dimension }\end{array}$ \\
\hline 2017 & [23] & $\begin{array}{c}\text { Transportation Research } \\
\text { Part E }\end{array}$ & $\begin{array}{c}\text { DEMATEL, ANP, Fuzzy } \\
\text { ER }\end{array}$ & $\begin{array}{c}\text { operation, financial, customer } \\
\text { satisfaction, logistics, } \\
\text { sustainability, safety }\end{array}$ \\
\hline 2017 & [108] & $\begin{array}{l}\text { Journal of Management, } \\
\text { Marketing and Logistics }\end{array}$ & TOPSIS & $\begin{array}{c}\text { infrastructure and } \\
\text { superstructure, operation }\end{array}$ \\
\hline 2017 & [109] & $\begin{array}{c}\text { Journal of Business } \\
\text { Management }\end{array}$ & & operation, financial \\
\hline 2017 & [110] & Marine Pollution Bulletin & $\begin{array}{l}\text { Semi-structured } \\
\text { interview }\end{array}$ & sustainability, financial \\
\hline 2017 & [47] & $\begin{array}{l}\text { Maritime Policy \& } \\
\text { Management }\end{array}$ & DEA & $\begin{array}{l}\text { sustainability, operation, } \\
\text { infrastructure and } \\
\text { superstructure }\end{array}$ \\
\hline 2018 & [111] & $\begin{array}{c}\text { International Journal of } \\
\text { Quality and Reliability } \\
\text { Management }\end{array}$ & $\begin{array}{l}\text { Sigma Value (SV), the } \\
\text { Process Capability } \\
\text { indices (PCIs), and the } \\
\text { Cost of Poor Quality } \\
\text { (COPQ) }\end{array}$ & operation, financial, safety \\
\hline 2018 & [112] & $\begin{array}{l}\text { Journal of ETA Maritime } \\
\text { Science }\end{array}$ & DEA & $\begin{array}{c}\text { infrastructure and } \\
\text { superstructure, operation }\end{array}$ \\
\hline 2018 & [113] & $\begin{array}{l}\text { The IUP Journal of Supply } \\
\text { Chain Management }\end{array}$ & $\begin{array}{c}\text { Importance } \\
\text { Performance Analysis } \\
\text { (IPA), Quality Function } \\
\text { Deployment (QFD) and } \\
\text { Interpretive Structural } \\
\text { Model (ISM) }\end{array}$ & $\begin{array}{l}\text { operation, customer } \\
\text { satisfaction }\end{array}$ \\
\hline 2018 & {$[14]$} & Logistics & Queue Analysis & $\begin{array}{l}\text { operation, logistics, } \\
\text { infrastructure and } \\
\text { superstructure }\end{array}$ \\
\hline 2018 & [36] & $\begin{array}{c}\text { Maritime Economics \& } \\
\text { Logistics }\end{array}$ & $\begin{array}{c}\text { Meta frontier analysis, } \\
\text { DEA, stochastic } \\
\text { frontier analysis }\end{array}$ & $\begin{array}{c}\text { operation, infrastructure and } \\
\text { superstructure }\end{array}$ \\
\hline 2018 & [114] & $\begin{array}{l}\text { Journal of Integrated } \\
\text { Coastal Zone } \\
\text { Management }\end{array}$ & Duncan Test & sustainability \\
\hline 2018 & {$[50]$} & $\begin{array}{l}\text { Production and } \\
\text { Operations Management } \\
\text { Society }\end{array}$ & Mathematical model & operation \\
\hline 2018 & [28] & Jurnal Teknik Industri & AHP & $\begin{array}{l}\text { financial, infrastructure and } \\
\text { superstructure, operation }\end{array}$ \\
\hline 2018 & [49] & $\begin{array}{l}\text { The Asian Journal of } \\
\text { Shipping and Logistics }\end{array}$ & $\begin{array}{c}\text { Factor analysis, } \\
\text { Regression Analysis }\end{array}$ & $\begin{array}{c}\text { financial, operation, customer } \\
\text { satisfaction }\end{array}$ \\
\hline 2018 & {$[40]$} & $\begin{array}{c}\text { Journal of Shipping and } \\
\text { Trade }\end{array}$ & Correlation Analysis & logistics, operation, financial \\
\hline 2019 & {$[44]$} & $\begin{array}{l}\text { Cogent Business \& } \\
\text { Management }\end{array}$ & $\begin{array}{l}\text { Structural equation } \\
\text { model }\end{array}$ & $\begin{array}{l}\text { infrastructure and } \\
\text { superstructure, financial, } \\
\text { operation }\end{array}$ \\
\hline
\end{tabular}


Table 1. Literature Table (Cont')

\begin{tabular}{|c|c|c|c|c|}
\hline Year & Reference & Journal & Method(s) & $\begin{array}{c}\text { Approached Performance } \\
\text { Dimension }\end{array}$ \\
\hline 2019 & [115] & $\begin{array}{l}\text { International Journal of } \\
\text { Information Management }\end{array}$ & $\begin{array}{c}\text { Correlation Analysis, } \\
\text { Regression Analysis }\end{array}$ & logistics, operation \\
\hline 2019 & [116] & $\begin{array}{c}\text { Transportation Research } \\
\text { Part D }\end{array}$ & Literature review & sustainability \\
\hline 2019 & [117] & $\begin{array}{l}\text { International Conference } \\
\text { on Engineering, Applied } \\
\text { Sciences and Technology }\end{array}$ & Regression Analysis & $\begin{array}{l}\text { operation, infrastructure and } \\
\text { superstructure }\end{array}$ \\
\hline 2019 & [118] & $\begin{array}{l}\text { Scientific Bulletin of } \\
\text { Naval Academy }\end{array}$ & Literature review & $\begin{array}{l}\text { operation, customer } \\
\text { satisfaction, logistics }\end{array}$ \\
\hline 2019 & [119] & Sustainability & Literature review & sustainability \\
\hline 2019 & {$[51]$} & Sustainability & AHP & $\begin{array}{l}\text { operation, financial, customer } \\
\text { satisfaction, sustainability }\end{array}$ \\
\hline 2019 & {$[120]$} & $\begin{array}{l}\text { Cogent Business \& } \\
\text { Management }\end{array}$ & $\begin{array}{c}\text { Exploratory Factor } \\
\text { analysis, One-Way } \\
\text { ANOVA }\end{array}$ & $\begin{array}{c}\text { sustainability, safety, financial, } \\
\text { operation }\end{array}$ \\
\hline 2019 & [6] & Complexity & Mathematical model & $\begin{array}{l}\text { operation, financial, } \\
\text { infrastructure and } \\
\text { superstructure, logistics, } \\
\text { sustainability }\end{array}$ \\
\hline 2019 & [122] & Transport Policy & $\begin{array}{c}\text { Importance- } \\
\text { Performance analysis }\end{array}$ & $\begin{array}{l}\text { operation, infrastructure and } \\
\text { superstructure, customer } \\
\text { satisfaction, financial, logistics }\end{array}$ \\
\hline 2019 & [123] & $\begin{array}{l}\text { AVRASYA Uluslararası } \\
\text { Araștırmalar Dergisi }\end{array}$ & DEMATEL & sustainability \\
\hline 2019 & [124] & $\begin{array}{c}\text { Maritime Economics \& } \\
\text { Logistics }\end{array}$ & $\begin{array}{l}\text { Panel Regression } \\
\text { Analysis }\end{array}$ & financial \\
\hline 2019 & {$[125]$} & $\begin{array}{l}\text { Journal of Yaşar } \\
\text { University }\end{array}$ & $\begin{array}{l}\text { AHP-TOPSIS hybrid } \\
\text { method }\end{array}$ & $\begin{array}{c}\text { financial, infrastructure and } \\
\text { superstructure, operation, } \\
\text { safety }\end{array}$ \\
\hline 2019 & [27] & Management Decision & Best-Worst method & $\begin{array}{l}\text { financial, infrastructure and } \\
\text { superstructure, operation, } \\
\text { customer satisfaction, logistics }\end{array}$ \\
\hline 2019 & {$[48]$} & $\begin{array}{l}\text { Maritime Policy \& } \\
\text { Management }\end{array}$ & $\begin{array}{l}\text { Exploratory Factor } \\
\text { analysis }\end{array}$ & $\begin{array}{l}\text { operation, financial, } \\
\text { infrastructure and } \\
\text { superstructure, logistics }\end{array}$ \\
\hline 2020 & [39] & $\begin{array}{l}\text { ISH Journal of Hydraulic } \\
\text { Engineering }\end{array}$ & Correlation Analysis & operation \\
\hline 2020 & [121] & $\begin{array}{c}\text { Maritime Economics \& } \\
\text { Logistics }\end{array}$ & $\begin{array}{c}\text { Discrete event } \\
\text { simulation model }\end{array}$ & operation, financial \\
\hline 2020 & [29] & Transport Policy & $\begin{array}{c}\text { T test, Multiple } \\
\text { Regression Analysis, } \\
\text { DEA }\end{array}$ & $\begin{array}{c}\text { operation, customer } \\
\text { satisfaction, infrastructure and } \\
\text { superstructure }\end{array}$ \\
\hline
\end{tabular}


As a result of the frequency analysis, it is seen that 25 articles were published between the years of 1995-2009, 40 articles between the years of 2010-2014, 59 articles between the years of 2015-2020. In the light of this information, 79.8 percent of these articles were published after the year 2010. This situation shows that recently, port performance studies have become a trend again in academic literature and there has been much more attention to it. When looking at the journals in which articles were published, 'Transportation Research' draws attention with 14 articles published on the subject, 'Maritime Policy \& Management' accompanied with 12 articles and 'Maritime Economics \& Logistics' followed up them with 9 articles. Besides, these three journals are followed by 'Research in Transportation Business and Management' with 5 articles, 'The Asian Journal of Shipping and Logistics' and 'Transport Policy' with 4 articles.

When we look at the statistical data on the most preferred methods in the articles (shown in Figure 1), it is seen that DEA comes to the forefront. Accordingly, while the number of articles employing DEA is 33, this number corresponds to approximately 27 percent of all articles. While 16 percent of the authors contribute to the port performance literature by producing review papers through a literature review, the number of articles that employed one of the MCDM methods is 15. Besides, while 12 studies measured the port performance by proposing a new mathematical model, 9 articles tried to develop a data collection tool related to port performance. The first study employed MCDM methods published in 2012. So, it is detected that the most frequently used method was MCDM methods after the year 2012. Studies that employed MCDM methods and DEA had made a significant contribution to the port performance concept in terms of monitoring the evolution of port performance indicators over the years. It is very difficult to develop a standard data collection tool to measure port performance due to various reasons such as the unique nature of each port type and constantly changing and evolving customer expectations. Perhaps, for this reason, the number of studies trying to combine all port performance criteria using factor analysis was limited to 9 .

Finally, the operational performance of the ports has been determined as the most discussed performance dimension in the articles. The operational dimension

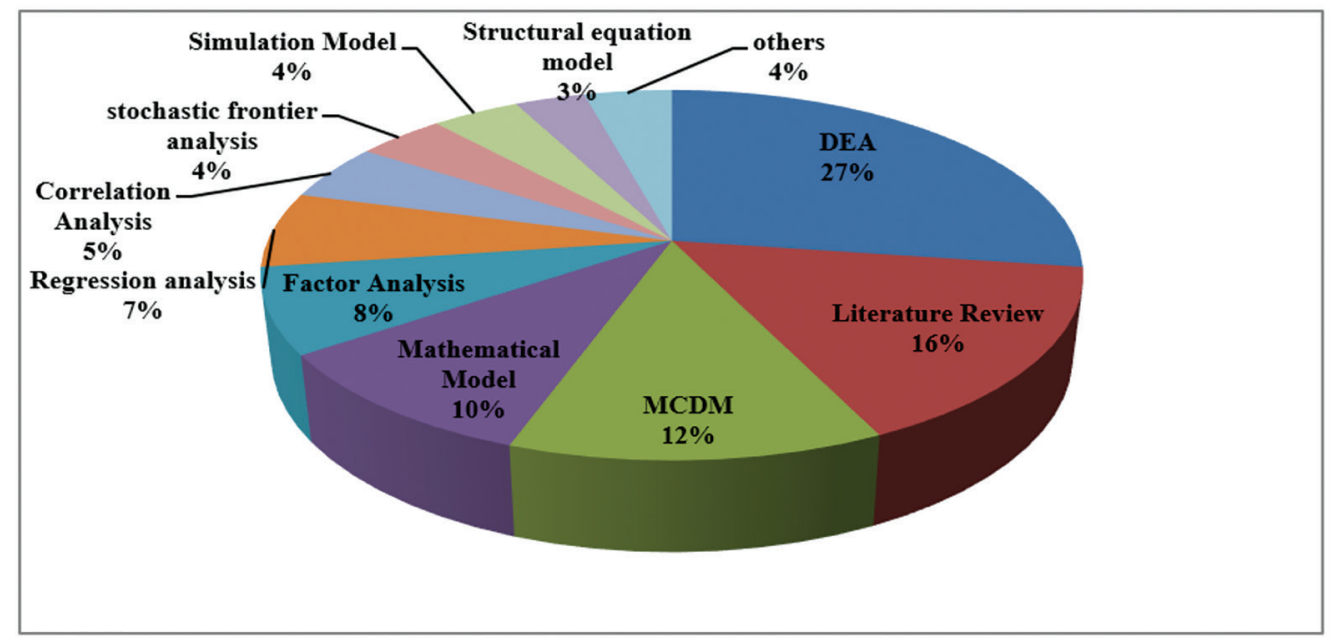

Figure 1. Employed Methods 
of port performance was approached in 105 different articles, which indicates that this dimension is examined in 86.78 percent of all articles. The financial (economic) dimension of port performance was discussed in 62 studies, and the sustainability dimension, which was trending especially after the year 2010, was handled in 39 studies. Lastly, the logistics dimension of port performance was examined in 22 studies. In the following section, the content of the studies on dimensions of the port performance will be analysed in detail.

\section{Dimensions of the Port Performance}

In this study contents of the 124 articles were analysed and port performance dimensions discussed in the articles were evaluated. As a result of the frequency analysis employed in this study, it was seen that port performance has operational, financial, sustainability, and logistics dimensions. In this section, indicators of each dimension to provide a measurement tool were presented.

\subsection{Operational Indicators}

According to Ducruet [126] and Mangan et al. [30], if the parameters of the port performance are constantly monitored, it becomes the standardized parameters of the port operations and these parameter values become the standard of the port [118]. Considering this thought, almost all studies on port performance in the literature either used the operation performance instead of the port performance or integrated an operational indicator into the port performance.

One of the most important indicators of operational performance is the speed concept, especially from a customer perspective. In this sense, Tongzon and Heng [54] and Kavakeb et al. [94] expressed the operational speed level in the ports as an important performance indicator, since the navigational costs of the ships are much lower than the costs during the time they are in the ports. Studies on improving port performance especially emphasize the concepts of efficiency and effectiveness so that port operations can be accelerated [106]. Herein, while traditional port performance indicators focus on specific efficiency criteria, what is expected from contemporary indicators is inclusive of all aspects of the operation and is consistent with the organization's strategies [68]. As almost all the studies analysing the operational performance of ports with DEA did, Lin and Tseng [15] and Ursavaş [89] used the number of calling ships and the loaded and unloaded container volume as outputs, in other words, performance indicators of the DEA model. Esmer [5], in addition to these indicators, approached such the indicators as the rate of the container loaded and unloaded, crane productivity, the automation level of the cranes, average container weight, ship turnaround time, total working time, stored container movement, labour force productivity, area utilization efficiency, equipment usage efficiency, costeffectiveness. Apart from these, Paing and Prabnasak [117] emphasized that such criteria as 'average waiting time while anchoring', 'average handling cargo tonnage per ship', 'berth occupancy rate', 'container dwell time', 'truck turnaround time' are used as performance indicators in literature. Finally, in the report published by UNCTAD [16], the operation performance of the ports was handled in two different ways: ship operation and cargo operation. Accordingly, while the report handled ship operation indicators with such criteria as "average waiting time (hours), average ship length (meters), average ship draft (meters), average ship gross tonnage"; cargo operation performance was analysed using such indicators as "average tonnage per ship call, cargo tonnage handled per 
working hour, the number of containers handled per hour, container dwell time (days), handled cargo per area (ton/ hectare), handled cargo ton per berth length".

\subsection{Financial Indicators}

Landlord ports can be influenced by three main factors: competition pressure for infrastructure investment, competition for land use, and financial pressure [109]. To eliminate or at least alleviate financial pressure, ports need to measure financial performance and check compliance with its targets. From a shareholder perspective, port authorities need to increase their net profitability, increase their overall market share, invest in new development projects, and in other words increase their financial performance [109]. The United Nations has accepted financial performance indicators, which measure the extent to which port authority converts capital and funds into performance, as one of the two most important criteria in measuring port performance [11]. The financial and operational performance of port authorities emerges as a result of managerial skills, and the financial performance of a port is of great importance to protect investments and to plan new projects in the future [109].

The financial performance of a port can generally be explained by the profitability of that port. Aguiar-Diaz et al. [124] while measuring the financial performance of Spanish ports, addressed the return of assets (ROA) of the ports as a criterion of performance. On the other hand, Wiegmans and Dekker [2] emphasized that two main indicators determine the financial performance of the ports and that they are sales and profitability. While Muangpan and Suthiwartnarueput [120] considered the unconsolidated financial situation of the port as a financial performance indicator, Roos and Neto [110] took into account financial investment requirement,
Bolevics [109] handled net profit, total market share, operating income, total debts, investment intangible fixed assets, Earnings Before Interest Taxes Depreciation and Amortization (EBITDA), Mickiene and Valioniene [38] addressed financial efficiency and financial autonomy, Aqmarina and Achjar [11] approached the rate of return and operating expenses as indicators of financial performance. Brooks and Pallis [58], who handled the financial performance of ports much more comprehensively, included financial indicators such as return on capital employed (ROCE), service revenues, service profitability, trade receivables, interest coverage ratio, terminal charges, ship charges, and these indicators' share in gross income and net profit.

\subsection{Sustainable Indicators}

While most of the studies related to port management are on the competitiveness or efficiency of the ports, undesirable variables such as $\mathrm{CO} 2$ emissions have been ignored in studies on port efficiency [83]. Ports have become a complex system due to factors such as the variety of cargo within them, their location close to the society, and responsibilities for the benefits of their stakeholders. For these reasons and considering today's climate conditions [76], proper management mentality against security and environmental risks within the port area has become very important [100]. To establish harmonious environmental protection and sustainable development, an effective environmental port management strategy needs to consider environmental hazards, mitigation options, forecasting methods, information about environmental indicators, and laws [92]. There are three critical processes to implement environmental management practices at the ports: cooperation with supply chain partners, environmentally friendly operations, and internal management 
support [67]. Air quality, greenhouse gas emissions, soil and ground resources, rubble, light and sound problems, water, and climate change can be counted among those that need to be improved environmentally to ensure port sustainability. For the economic dimension, indicators such as the benefits of the port users, fair competition, employment, economic development, and tourism and port investment should be taken into account [37]. Environmental performance indicators have tasks such as providing information about environmental problems, supporting development policies and determining priorities, monitoring the effects of policies, pursuing environmental targets, comparing environmental performance over time, and attracting the attention of the society [61]. The social dimension, especially human resources, had been seen as independent variables or input elements. The safety aspect of social sustainability came to the forefront of the literature. The issue of ensuring a safe operation has gained currency lately in the literature and studies conclude that appropriate working conditions have increased labour efficiency and thus the operational performance of the port [100]. For this reason, the safety of the port area has started to be associated with the concepts of effectiveness and competitiveness [68]. Other important results of ensuring safe operation in the port area are the hiring of qualified workers, employing them longterm, and minimizing the economic and social losses of accidents. Recognition of a port as a safe port has a meaning much more for the related unit than business units serving in other industries [45]. Because of the port becomes inoperable due to emerging unsafe situations in the port area, it will have negative results both socially and economically. To take precautions against unsafe situations, it is very important to know what these situations are. Darbra and Casal [127] stated that accidents in ports are mostly occurred during the manoeuvring of the ships, while Yip [128] revealed that most of the accidents in the port area occurred due to the ships crashing into the dock [100]. Unlike, Mollaoğlu et al. [129] grouped the factors that caused the accident in the port area as labour induced, vehicle and equipment induced, facility induced, lack of coordination induced. Accordingly, overconfidence and disengagement behaviour, which are among the labour induced factors, have been identified as the most leading reason for the accidents in the ports.

Lim et al. [116] had not encountered in the literature any studies that are concerned only with the social or economic dimension of sustainability. The general trend in the related literature is that the concepts of sustainable port performance and environmental port performance are interwoven. The first time, Darbra et al. [53] introduced the project, which expresses environmentally friendly practices in the ports, named as the 'Self Diagnosis Method' carried out by ESPO. In this project, criteria such as "air quality, dredging activities, dust management, energy usage, loss of habitat, health and safety management, noise management, soil pollution, waste management and water quality" were used as indicators of sustainable port performance. Saengsupavanich et al. [61] addressed both countable and uncountable criteria such as the number of ISO 14001 Environmental Management System certified facilities and terminals, the number of environmental complaints, the number of fuel/chemical leakage incidents, water quality around the port, penalties imposed on non-observant operators, number of environmental department employees, number of ships inspected annually in the port, environmental expenditures, taxes and allowances, accessibility to the emergency plan, frequency of training, 
knowledge level of employees on the port state controls, protection of environmental policies, as an indicator of environmental performance. Apart from these, Park and Yeo [73] analysed such indicators as "alternative fuel usage, incentives to reduce pollution, renewable energy usage, development of the breakwater system to revive the dock, resource recycling in the port area, mode change to prevent traffic congestion, artificial sand pile and wetland creation", while they were evaluating the environmental performance of Korean ports. Many new criteria have been added to these sustainable performance indicators over the years: cold ironing (onshore power supply to ships) [76], CO2 emission control [83], odour management [92], water consumption level, and tariff discount to green ships [100], green material usage in the port building process and attending related conferences [37], environmental costs [110], area consumption level [119]. On the other hand, in the social dimension, Antao et al. [100] used such indicators as "the number of off days due to accident, the accident frequency rate, the number of fatal work accidents, the total number of work accidents, the degree of accident severity, the number of absenteeism due to accident or illness, the number of seaward accidents, the number of ships crashes into the dock, the number of near-miss, the number of leakage incidents, the number of fires or explosions" to measure the safety performance of the ports. Brooks [57] handled frequency of accidents, Woo et al. [68] approached compliance with regulations, the number of accidents and the number of prevented accidents, Brooks and Schellinck [79] focused on prejudicial to cargo incidents, Ha et al. [23] used the determination of restricted areas, formal safety training practices, number of adequate observation and threat awareness as indicators that determine whether ports are safe or not.

\subsection{Logistics Indicators}

For the logistics world, ports are an important nodal point so that they provide intermodal and multimodal transportation services and operate as logistics centres for cargo and passengers [10]. Today, almost all the services provided by logistics companies are expected from the ports by its customers. For that reason alone, ports should cooperate with supply chain partners to provide value-added services to their stakeholders [49]. Among the advantages that a port cooperates with logistics service providers, not only does it increase the value of the relevant port supply chain, but also decreases the value of competing for port supply chains [101]. Through this, many companies are involved in logistics and supply chain integration throughout the port and around the ports [10]. Due to the changing logistics environment, ports should carefully monitor changes and produce strategies accordingly [68].

The logistics performance of the ports is often based on efficiency and utility measurements. Bichou [130] stated that since ports have used their facilities for logistics, production, and economic activities, new port performance indicators are needed [14]. Accordingly, many indicators determine the logistics performance of the ports. These indicators were processed in the academic literature in a way that will differ according to the years, in other words, they were shaped according to the market situation. Bichou and Gray [10] have identified processes such as logistics integration, benchmarking, logistics channel design, value-added services, customer service as indicators of a port's logistics performance. Woo et al. [68] added indicators such as service quality, customer orientation level, auxiliary service prices, intermodal cargoes' waiting and working times, to the literature. Seo et al. [101] used the logistics performance indicators of the ports such as convenience to the 
port users, safety, and security throughout the hinterland, and reliability. Han [49] considered performance dimensions such as cost performance, quality performance, and responsiveness as indicators of the logistics performance of the ports. Finally, Ha et al. [122] have taken into account the level of intermodal transportation systems and value-added services as an indicator of logistics integrations of container terminals.

\section{Discussion}

When the literature is analysed, it is seen that some studies have made a literature review regarding the port performance and as a result of their analysis, they brought different perspectives to the port performance concept. These studies were analysed in detail and detached aspects of these studies from our study were revealed. Thus, the originality of this study and its contribution to the literature was tried to be revealed. Langenus and Dooms [13] evaluated 74 articles in literature and drew attention to the gap that is less concern on industry specific ports' performance. And the authors proposed that new developments such as the container revolution, big data analytics, knowledge transparency, which affect port performance, should be assessed. Lim et al. [116] reviewed 21 articles focused on the sustainability performance of ports and proposed that social indicators of port performance should be revealed. In our research, it is determined that 8 social indicators revealed in that study used generally as input or independent variable to assess ports' overall performance. Somensi et al. [26] analysed 37 articles in literature and suggested that it should be evaluated whether port management activities contribute to port performance. Similarly, Vieira et al. [86] advocated that there is a research gap in the relationship between port governance and performance. On the other hand, our research suggested a more descriptive approach for collecting data and measuring port performance. Dutra et al. [1] handled 23 articles and remarked that most of the studies are out of interacting with port managers. Unlike, we think that stakeholders of ports should evaluate service quality they receive and thus, port performance would show up.

No other study focusing on the dimensions of port performance was found among the descriptive studies in the literature. On the other hand, it was observed that the empirical studies did not analyse cases by combining the dimensions of the port performance or by separating the related dimensions. Since performance dimensions were thought to have a natural relationship with each other or no measurement model seems to allow this separation. For instance, Brooks and Schellinck [79] asked customers of US and Canadian ports to evaluate the five-year performance of the most frequent port they work with. While they were evaluating these ports' performance, they did measure operational, safety, and logistics performance, but did not take into account financial and sustainable dimensions. On the other hand, most of studies had used operational indicators to evaluate overall performance of ports [42][7][55][15][43] [8][32][72][35][80][21][85][94][95][99] [24][25][107][108][36][39][50]. However, the originality of this study comes from this point. Our research suggests that analyses on port performance should be made by separating its dimensions from each other. After this separation, an analysis of the preferred dimension(s) should be carried out.

\section{Conclusion}

Ports are more than just a meeting point for carriers and shippers today but are the nodes of global trade and produce valueadded services for many stakeholders. So, the concept of port performance has 
changed greatly over the years and the performance perception of each port stakeholder has differed from each other. For example, while operational quality in the terminal area is perceived as a high performance by shippers or carriers, on the other hand, the legislative bodies or local community perceive efficient sustainability applications as high performance or logistics service providers care about hinterland connection quality more. At this point, the perception of the shippers, the port authority, the company that provides towage and pilotage service, etc. can differ from each other. For this reason, it is very difficult to establish a standard performance measurement. Besides, considering the competition between the ports outside the port area, it is also important to know which performance dimension is desired to measure.

In order to overcome the challenges of standardising port performance measurement, different perceptions of the stakeholders should be gathered and obtained an overall score or should be exactly separated from each other. So as a contribution of this study, dimensions of the port performance were revealed to bring a new perception to the port performance concept. Moreover, indicators of each dimension were developed for empirical analyses. Thus, different aspects of port performance will be determined and also assessed. Maybe the contribution level of each aspect to overall performance can be evaluated.

For further studies, it would be appropriate to develop a measurement on in which dimension of the port performance is desired to be examined. Although corporate social responsibility (CSR) in ports had been studied many times before, the effectiveness or efficiency of CSR activities was not analysed in the literature. Thus, performance criteria regarding ports' CSR practices can be developed. Most of the studies assessed the operational performance of the ports had seen human resources as an independent variable or input factor to achieve high performance. However, factors that affect human resource quality can be studied. In this way, in-depth analysis of operational performance can be presented.

\section{References}

[1] Dutra, A., Ripoll-Feliu, V. M., Fillol, A. G., Ensslin, S. R., \& Ensslin, L. (2015). The construction of knowledge from the scientific literature about the theme seaport performance evaluation. International Journal of Productivity and Performance Management, 64(2), 243-269.

[2] Wiegmans, B., \& Dekker, S. (2016). Benchmarking deep-sea port performance in the Hamburg-Le Havre range. Benchmarking: An International Journal, 23(1), 96-112.

[3] Caldeirinha, V. R., \& Felício, J. A. (2014). The relationship between 'positionport', 'hard-port' and 'soft-port' characteristics and port performance: conceptual models. Maritime Policy \& Management, 41(6), 528-559.

[4] Ha, M. H., \& Yang, Z. (2017). Comparative analysis of port performance indicators: Independency and interdependency. Transportation Research Part A: Policy and Practice, 103, 264-278.

[5] Esmer, S. (2008). Performance measurements of container terminal operations. Dokuz Eylül Üniversitesi Sosyal Bilimler Enstitüsü Dergisi, 10(1), 238-255.

[6] Hossain, N. U. I., Nur, F., Jaradat, R., Hosseini, S., Marufuzzaman, M., Puryear, S. M., \& Buchanan, R. K. (2019). Metrics for assessing overall performance of inland waterway ports: A bayesian network based approach. Complexity, 1-17. 
[7] Cullinane, K., Song, D. W., Ji, P., \& Wang, T. F. (2004). An application of DEA windows analysis to container port production efficiency. Review of network Economics, 3(2), 184-206.

[8] Chudasama, K. M. (2009). Performance appraisal of Indian major ports using port ranking model. IUP Journal of Infrastructure, 7(1), 7-21.

[9] Schellinck, T., \& Brooks, M. R. (2014). Improving port effectiveness through determinance/performance gap analysis. Maritime Policy \& Management, 41(4), 328-345.

[10] Bichou, K., \& Gray, R. (2004). A logistics and supply chain management approach to port performance measurement. Maritime Policy \& Management, 31(1), 47-67.

[11] Aqmarina, A., \& Achjar, N. (2018). Determinants of port performancecase study of 4 main ports in Indonesia (2005-2015). Economics and Finance in Indonesia, 63(2), 176185.

[12] Ateş, A., \& Esmer, S. (2014). Farklı yöntemler ile türk konteyner limanlarının verimliliği. Verimlilik Dergisi, (1), 61-76.

[13] Langenus, M., \& Dooms, M. (2015). Port industry performance management: A meso-level gap in literature and practice?. International Journal of Logistics Research and Applications, 18(3), 251-275.

[14] Onwuegbuchunam, D. E. (2018). Assessing port governance, devolution and terminal performance in Nigeria. Logistics, 2(1), 1-16.

[15] Lin, L. C., \& Tseng, C. C. (2007). Operational performance evaluation of major container ports in the AsiaPacific region. Maritime Policy \& Management, 34(6), 535-551.

[16] UNCTAD (2016). Port Management Series: Volume 4 Port Performance. UNCTAD Publishing: Geneva.
[17] Tongzon, J. L. (1995). Determinants of port performance and efficiency. Transportation Research Part A: Policy and Practice, 29(3), 245-252.

[18] Martinez-Budria, E., Diaz-Armas, R., Navarro-Ibanez, M., \& Ravelo-Mesa, T. (1999). A study of the efficiency of Spanish port authorities using data envelopment analysis. International Journal of Transport Economics/Rivista internazionale di economia dei trasporti, 237-253.

[19] Bichou, K. (2006). Review of port performance approaches and a supply chain framework to port performance benchmarking. Research in Transportation Economics, 17, 567-598.

[20] Cullinane, K., Wang, T. F., Song, D. W., \& Ji, P. (2006). The technical efficiency of container ports: Comparing data envelopment analysis and stochastic frontier analysis. Transportation Research Part A: Policy and Practice, 40(4), 354-374.

[21] Wanke, P. F. (2013). Physical infrastructure and shipment consolidation efficiency drivers in Brazilian ports: A two-stage networkDEA approach. Transport Policy, 29, 145153.

[22] Talley, W. K. (2006). Port performance: an economics perspective. Research in Transportation Economics, 17, 499-516.

[23] Ha, M. H., Yang, Z., Notteboom, T., Ng, A. K., \& Heo, M. W. (2017). Revisiting port performance measurement: A hybrid multi-stakeholder framework for the modelling of port performance indicators. Transportation Research Part E: Logistics and Transportation Review, 103, 1-16.

[24] Suárez-Alemán, A., Sarriera, J. M., Serebrisky, T., \& Trujillo, L. (2016). When it comes to container port efficiency, are all developing regions equal?. Transportation Research Part A: Policy and Practice, 86, 56-77. 
[25] Estrada, M. A. R., Jenatabadi, H. S., \& Chin, A. T. (2017). Measuring ports efficiency under the application of PEP-model. Procedia Computer Science, 104, 205-212.

[26] Somensi, K., Ensslin, S., Dutra, A., Ensslin,L.,Ripoll-Feliu,V.M., \&Dezem, V. (2017). Knowledge construction about port performance evaluation: An international literature analysis. Intangible Capital, 13(4), 720-744.

[27] Rezaei, J., van Wulfften Palthe, L., Tavasszy, L., Wiegmans, B., \& van der Laan, F. (2019). Port performance measurement in the context of port choice: an MCDA approach. Management Decision, 57(2), 396417.

[28] Sentia, P. D., Ramadani, R., \& Zuhri, S. (2018). Logistic performance determination on the arrival of ship container. Jurnal Teknik Industri, 20(1), 59-64.

[29] Chen, Y., Yang, D., Lian, P., Wan, Z., \& Yang, Y. (2020). Will structureenvironment-fit result in better port performance?-An empirical test on the validity of Matching Framework Theory. Transport Policy, 86, 23-33.

[30] Feng, M., Mangan, J., \& Lalwani, C. (2012).Comparing portperformance: Western European versus Eastern Asian ports. International Journal of Physical Distribution \& Logistics Management, 42 (5), 490-512.

[31] Kang, D. J., \& Woo, S. H. (2017). Liner shipping networks, port characteristics and the impact on port performance. Maritime Economics \& Logistics, 19(2), 274295.

[32] Wu, J., Yan, H., \& Liu, J. (2009). Groups in DEA based cross-evaluation: An application to Asian container ports. Maritime Policy \& Management, 36(6), 545-558.
[33] Tongzon, J. (2001). Efficiency measurement of selected Australian and other international ports using data envelopment analysis. Transportation Research Part A: Policy and Practice, 35(2), 107-122.

[34] Al-Eraqi, A. S., Mustafa, A., \& Khader, A. T. (2010). An extended DEA windows analysis: Middle East and East African seaports. Journal of Economic Studies, 37(2), 208-218.

[35] Ferrari, C., Puliafito, P. P., \& Tei, A. (2013). Performance and quality indexes in the evaluation of the terminal activity: A dynamic approach. Research in Transportation Business \& Management, 8, 77-86.

[36] Nguyen, H. O., Nghiem, H. S., \& Chang, Y. T. (2018). A regional perspective of port performance using metafrontier analysis: the case study of Vietnamese ports. Maritime Economics \& Logistics, 20(1), 112-130.

[37] Lu, C. S., Shang, K. C., \& Lin, C. C. (2016). Examining sustainability performance at ports: port managers' perspectives on developing sustainable supply chains. Maritime Policy \& Management, 43(8), 909927.

[38] Mickiene, R., \& Valioniene, E. (2017). Evaluation of the interaction between the state seaport governance model and port performance indicators. Forum Scientiae Oeconomia, 5 (3), 27-43.

[39] Shetty, D. K., \& Dwarakish, G. S. (2020). Measuring port performance and productivity. ISH Journal of Hydraulic Engineering, 26(2), 221227.

[40] Mlimbila, J., \& Mbamba, U. O. (2018). The role of information systems usage in enhancing port logistics performance: evidence from the Dar Es Salaam port, Tanzania. Journal of Shipping and Trade, 3(10), 1-20. 
[41] Park, R. K., \& De, P. (2015). An alternative approach to efficiency measurement of seaports. In Port Management (pp. 273-292). Palgrave Macmillan, London.

[42] Itoh, H. (2002). Efficiency changes at major container ports in Japan: a window application of Data Envelopment Analysis. Review of urban \& regional development studies, 14(2), 133-152.

[43] Al-Eraqi, A. S., Mustafa, A., Khader, A. T., \& Barros, C. P. (2008). Efficiency of Middle Eastern and East African seaports: application of DEA using window analysis. European journal of scientific research, 23(4), 597612.

[44] Sirajuddin, Zagloel, T. Y., \& Sunaryo. (2019). Effect of strategic alliance based on port characteristic and integrated global supply chain for enhancing industrial port performance. Cogent Business \& Management, 6(1567893), 1-14.

[45] Pak, J. Y., Thai, V. V., \& Yeo, G. T. (2015). Fuzzy MCDM approach for evaluating intangible resources affecting port service quality. The Asian Journal of Shipping and Logistics, 31(4), 459-468.

[46] Cullinane, K., \& Wang, T. (2010). The efficiency analysis of container port production using DEA panel data approaches. OR spectrum, 32(3), 717-738.

[47] Cheon, S., Maltz, A., \& Dooley, K. (2017). The link between economic and environmental performance of the top 10 US ports. Maritime Policy \& Management, 44(2), 227-247.

[48] Jeevan, J., Chen, S. L., \& Cahoon, S. (2019). The impact of dry port operations on container seaports competitiveness. Maritime Policy \& Management, 46(1), 4-23.
[49] Han, C. H. (2018). Assessing the impacts of port supply chain integration on port performance. The Asian Journal of Shipping and Logistics, 34(2), 129-135.

[50] Wang, Z., Yao, D. Q., Yue, X., \& Liu, J. J. (2018). Impact of IT capability on the performance of port operation. Production and Operations Management, 27(11), 1996-2009.

[51] Hervás-Peralta, M., Poveda-Reyes, S., Molero, G. D., Santarremigia, F. E., \& Pastor-Ferrando, J. P. (2019). Improving the performance of dry and maritime ports by increasing knowledge about the most relevant functionalities of the Terminal Operating System (TOS). Sustainability, 11(6), 1648.

[52] Teng, J. Y., Huang, W. C., \& Huang, M. J. (2004). Multicriteria evaluation for port competitiveness of eight East Asian container ports. Journal of Marine Science and Technology, 12(4), 256-264.

[53] Darbra, R. M., Ronza, A., Casal, J., Stojanovic, T. A., \& Wooldridge, C. (2004). The Self Diagnosis Method: A new methodology to assess environmental management in sea ports. Marine Pollution Bulletin, 48(5-6), 420-428.

[54] Tongzon, J., \& Heng, W. (2005). Port privatization, efficiency and competitiveness: Some empirical evidence from container ports (terminals). Transportation Research Part A: Policy and Practice, 39(5), 405-424.

[55] Cullinane, K. P., \& Wang, T. F. (2006). The efficiency of European container ports: A cross-sectional data envelopment analysis. International Journal of Logistics: Research and Applications, 9(1), 19-31. 
[56] Chou, C. C. (2007). A fuzzy MCDM method for solving marine transshipment container port selection problems. Applied Mathematics and Computation, 186(1), 435-444.

[57] Brooks, M. R. (2007). Issues in measuring port devolution program performance: a managerial perspective. Research in transportation Economics, 17, 599629.

[58] Brooks, M. R., \& Pallis, A. A. (2008). Assessing port governance models: process and performance components. Maritime Policy \& Management, 35(4), 411-432.

[59] Lam, J. S. L., \& Yap, W. Y. (2008). Competition for transhipment containers by major ports in Southeast Asia: slot capacity analysis. Maritime Policy \& Management, 35(1), 89-101.

[60] Yeo, G. T., Roe, M., \& Dinwoodie, J. (2008). Evaluating the competitiveness of container ports in Korea and China. Transportation Research Part A: Policy and Practice, 42(6), 910-921.

[61] Saengsupavanich, C., Coowanitwong, N.,Gallardo, W.G., \& Lertsuchatavanich, C. (2009). Environmental performance evaluation of an industrial port and estate: IS014001, port state controlderived indicators. Journal of Cleaner Production, 17(2), 154-161.

[62] Wu, J., Yan, H., \& Liu, J. (2010). DEA models for identifying sensitive performance measures in container port evaluation. Maritime Economics \& Logistics, 12(3), 215-236.

[63] Wu, J., Liang, L., \& Song, M. (2010). Performance based clustering for benchmarking of container ports: an application of DEA and cluster analysis technique. International Journal of Computational Intelligence Systems, 3(6), 709-722.
[64] Simões, P., \& Marques, R. C. (2010). Seaport performance analysis using robust non-parametric efficiency estimators. Transportation Planning and Technology, 33(5), 435-451.

[65] Dragovic, B., \& Zrnic, N. D. (2011). A queuing model study of port performance evolution. Analele Universitatii" Eftimie Murgu" Resita Fascicola de Inginerie, 2(18), 65-76.

[66] Ding, J. F., \& Chou, C. C. (2011). A fuzzy MCDM model of service performance for container ports. Scientific research and Essays, 6(3), 559-566.

[67] Venus Lun, Y. H. (2011). Green management practices and firm performance: A case of container terminal operations. Resources, Conservation and Recycling, 55(6), 559-566.

[68] Woo, S. H., Pettit, S., \& Beresford, A. K. (2011). Port evolution and performance in changing logistics environments. Maritime Economics \& Logistics, 13(3), 250-277.

[69] Onut, S., Tuzkaya, U. R., \& Torun, E. (2011). Selecting container port via a fuzzy ANP-based approach: A case study in the Marmara Region, Turkey. Transport Policy, 18(1), 182-193.

[70] Arlı, E. (2012). Konumlandırma stratejilerinin işletme performansı ile ilişkisi: Liman işletmeciliğinde bir uygulama. Journal of Management \& Economics, 19(2), 99-121.

[71] Madeira, A. G. J., Cardoso, M. M. J., Belderrain, M. C. N., Correia, A. R., \& Schwanz, S. H. (2012). Multicriteria and multivariate analysis for port performance evaluation. International Journal of Production Economics, 140(1), 450-456.

[72] Almaz, O. A., \& Altıok, T. (2012). Simulation modeling of the vessel traffic in Delaware River: Impact of deepening on port performance. Simulation Modelling Practice and Theory, 22, 146-165. 
[73] Park, J. Y., \& Yeo, G. T. (2012). An evaluation of greenness of major Korean ports: A fuzzy set approach. The Asian Journal of Shipping and Logistics, 28(1), 67-82.

[74] Dias, J. C. Q., Azevedo, S. G., Ferreira, J. M., \& Palma, S. F. (2012). Seaport performance comparison using data envelopment analysis: the case of Iberian container terminals. International Journal of Business Performance Management, 13(3-4), 426-449.

[75] Gong, S. X., Cullinane, K., \& Firth, M. (2012). The impact of airport and seaport privatization on efficiency and performance: A review of the international evidence and implications for developing countries. Transport Policy, 24, 37-47.

[76] Lirn, T. C., Wu, Y. C. J., \& Chen, Y. J. (2013). Green performance criteria for sustainable ports in Asia. International Journal of Physical Distribution \& Logistics Management, 43 (5/6), 427-451.

[77] Yang, Y. C., \& Chang, W. M. (2013). Impacts of electric rubber-tired gantries on green port performance. Research in Transportation Business \& Management, 8, 67-76.

[78] De Langen, P. W., \& Sharypova, K. (2013). Intermodal connectivity as a port performance indicator. Research in Transportation Business \& Management, 8, 97-102.

[79] Brooks, M. R., \& Schellinck, T. (2013). Measuring port effectiveness in user service delivery: What really determines users' evaluations of port service delivery? Research in Transportation Business \& Management, 8, 87-96.

[80] Bergantino, A. S., Musso, E., \& Porcelli, F. (2013). Port management performance and contextual variables: Which relationship? Methodological and empirical issues. Research in Transportation Business \& Management, 8, 39-49.
[81] Öztürk, E., Mesci, M., \& Kılınç, İ. (2013). Yenilik faaliyetlerinin işletme performansına etkisi: yat limanları üzerine bir değerlendirme. Girișimcilik ve Kalkınma Dergisi, 8 (2), 97-118.

[82] Woo, S. H., Pettit, S. J., \& Beresford, A. K. (2013). An assessment of the integration of seaports into supply chains using a structural equation model. Supply Chain Management: An International Journal, 18 (3), 235252.

[83] Chang, Y. T. (2013). Environmental efficiency of ports: a data envelopment analysis approach. Maritime Policy \& Management, 40(5), 467-478.

[84] Lam, J. S. L., \& Song, D. W. (2013). Seaport network performance measurement in the context of global freight supply chains. Polish Maritime Research, 20(Special Issue), 47-54.

[85] Schøyen, H., \& Odeck, J. (2013). The technical efficiency of Norwegian container ports: A comparison to some Nordic and UK container ports using Data Envelopment Analysis (DEA). Maritime Economics \& Logistics, 15(2), 197-221.

[86] Vieira, G. B. B., Kliemann Neto, F. J., \& Amaral, F. G. (2014). Governance, governance models and port performance: A systematic review. Transport Reviews, 34(5), 645-662.

[87] Güner, S., Coşkun, E., \& Taşkın, K. (2014). Liman özelleştirmelerinin operasyonel etkinlik üzerindeki etkisi: Türk limanları üzerinde dönemsel bir çalışma. İstanbul Üniversitesi İşletme Fakültesi Dergisi, 43(2), 218-236.

[88] Talley, W. K., Ng, M., \& Marsillac, E. (2014). Port service chains and port performance evaluation. Transportation Research Part E: Logistics and Transportation Review, 69, 236-247. 
[89] Ursavaş, E. (2014). A decision support system for quayside operations in a container terminal. Decision Support Systems, 59, 312-324.

[90] Rios, A. M. C., \& de Sousa Ramos, F. (2014). Cluster analysis of the competitiveness of container ports in Brazil. Transportation Research Part A: Policy and Practice, 69, 423-431.

[91] Li, J., \& Jiang, B. (2014). Cooperation performance evaluation between seaport and dry port; case of Qingdao port and Xi'an port. International Journal of e-Navigation and Maritime Economy, 1, 99-109.

[92] Puig, M., Wooldridge, C., \& Darbra, R. M. (2014).Identification and selection of environmental performance indicators for sustainable port development. Marine Pollution Bulletin, 81(1), 124-130.

[93] Shaheen, A., \& El-All, H. M. A. (2014). The competitive advantage of seaports and applied to the east port said-port said. International Journal of Research in Applied, Natural and Social Sciences, 2 (11), 111-120.

[94] Kavakeb, S., Nguyen, T. T., McGinley, K., Yang, Z., Jenkinson, I., \& Murray, R. (2015). Green vehicle technology to enhance the performance of a European port: a simulation model with a cost-benefit approach. Transportation Research Part C: Emerging Technologies, 60, 169188.

[95] Wiegmans, B., Witte, P. A., \& Spit, T. J. M. (2015). Inland port performance: a statistical analysis of Dutch inland ports. Current Practices in Transport: Appraisal Methods, Policies and Models, 8, 145-154.

[96] Güner, S. (2015). Liman etkinliği ölçümünde iki aşamalı bir model önerisi ve türk limanları üzerinde bir uygulama. Alphanumeric Journal, 3(2), 99-106.
[97] Oliveira, G. F. D., \& Cariou, P. (2015). The impact of competition on container port (in) efficiency. Transportation Research Part A: Policy and Practice, 78, 124-133.

[98] Ergin, A., Eker, İ., \& Alkan, G. (2015). Selection of container port using ELECTRE technique. International Journal of Operations and Logistics Management, 4(4), 268-275.

[99] Chen, L., Zhang, D., Ma, X., Wang, L., Li, S., Wu, Z., \& Pan, G. (2016). Container port performance measurement and comparisonleveragingshipGPStraces and maritime open data. IEEE Transactions on Intelligent Transportation Systems, 17(5), 1227-1242.

[100] Antão, P., Calderón, M., Puig, M., Michail, A., Wooldridge, C., \& Darbra, R. M. (2016). Identification of occupational health, safety, security (OHSS) and environmental performance indicators in port areas. Safety Science, 85, 266-275.

[101] Seo, Y. J., Dinwoodie, J., \& Roe, M. (2016). The influence of supply chain collaboration on collaborative advantage and port performance in maritime logistics. International Journal of Logistics Research and Applications, 19(6), 562-582.

[102] Chen, Z., \& Pak, M. (2017). A Delphi analysis on green performance evaluation indices for ports in China. Maritime Policy \& Management, 44(5), 537-550.

[103] Ha, M. H., Yang, Z., \& Heo, M. W. (2017). A new hybrid decision making framework for prioritising port performance improvement strategies. The Asian Journal of Shipping and Logistics, 33(3), 105-116.

[104] Daldır, I. \& Tosun, Ö. (2017). Comparison of the port authority's efficiency in Turkey. Journal of Management, Marketing and Logistics, 4 (2), 152-158. 
[105]Cheon, S., Song, D. W., \& Park, S. (2018). Does more competition result in better port performance? Maritime Economics \& Logistics, 20(3), 433-455.

[106]Sridi, I., Bouguerra, H., \& Benammou, S. (2017). Performance of the Tunisian port system. LOGISTIQUA, 88-93.

[107]Burhani, J. T., Zukhruf, F., \& Frazila, R. B. (2017). Port performance evaluation tool based on microsimulation model. MATEC Web of Conferences, 101, 1-5.

[108]Acer, A., \& Yangınlar, G. (2017). The determination of Turkish container ports performance with TOPSIS multiple criteria decision making method. Journal of Management Marketing and Logistics, 4(2), 67-75.

[109]Bolevics, V. (2017). The Impact of Governance on the Efficiency of the Baltic States' Major Ports. Journal of Business Management, 14, 7-26.

[110]Roos, E. C., \& Neto, F. J. K. (2017). Tools for evaluating environmental performance at Brazilian public ports: Analysis and proposal. Marine Pollution Bulletin, 115(1-2), 211-216.

[111]Ridwan, A., \& Noche, B. (2018). Model of the port performance metrics in ports by integration six sigma and system dynamics. International Journal of Quality \& Reliability Management, 35 (1), 82-108.

[112]Sağlam, B. B., Açık, A., \& Ertürk, E. (2018). Evaluation of investment impact on port efficiency: Berthing time difference as a performance indicator. Journal of ETA Maritime Science, 6(1), 37-46.

[113]Manikandan, M., \&Chidambaranathan, S. (2018). A conceptual hybrid model to improve the performance of port supply chain using importance performance analysis, quality function deployment and interpretive structural modeling. IUP Journal of Supply Chain Management, 15(2), 7-20.
[114]Rocha, C. H., Silva, G. L., \& de Abreu, L. M. (2018). Analysis of the evolution of Brazilian Ports' environmental performances. Journal of Integrated Coastal Zone Management, 18(2), 103-109.

[115]Aloini, D., Benevento, E., Stefanini, A. \& Zerbino, P. (2020). Process fragmentation and port performance: merging SNA and text mining. International Journal of Information Management, 51, 1-14.

[116]Lim, S., Pettit, S., Abouarghoub, W., \& Beresford, A. (2019). Port sustainability and performance: A systematic literature review. Transportation Research Part D: Transport and Environment, 72, 4764.

[117]Paing, W. P., \& Prabnasak, J. (2019). Determinants of Port PerformanceCase Study of Five Major Container Ports in Myanmar. IOP Conf. Ser.: Mater. Sci. Eng., 639(1), 1-8.

[118]Florin, N., Cotorcea, A., Filip, A., Bucur, M., \& Buciu, A. (2019). Performance measurement of the port logistics system. Scientific Bulletin" Mircea cel Batran" Naval Academy, 22(1), 1-11.

[119]Hui, F. K. P., Aye, L., \& Duffield, C. F. (2019). Engaging employees with good sustainability: key performance indicators for dry ports. Sustainability, 11(10), 1-11.

[120]Muangpan, T., \& Suthiwartnarueput, K. (2019). Key performance indicators of sustainable port: Case study of the eastern economic corridor in Thailand. Cogent Business \& Management, 6, 1-18.

[121]Musso, E., \& Sciomachen, A. (2020). Impact of megaships on the performance of port container terminals. Maritime Economics \& Logistics, 22, 432-445. 
[122]Ha, M. H., Yang, Z., \& Lam, J. S. L. (2019). Port performance in container transport logistics: A multistakeholder perspective. Transport Policy, 73, 25-40.

[123]Korucuk, S., \& Memiş, S. (2019). Yeşil liman uygulamaları performans kriterlerinin DEMATEL yöntemi ile önceliklendirilmesi: İstanbul örneği. Avrasya Uluslararası Araştırmalar Dergisi, 7(16), 134-148.

[124]Aguiar-Diaz, I., Ruiz-Mallorquí, M. V., \& Trujillo, L. (2019). Ownership structure and financial performance of Spanish port service companies. Maritime Economics \& Logistics, 1-25.

[125]Görçün, Ö. F. (2019). An Integrated AHP-TOPSIS Approach for Terminal Selection Problems in the Logistics Management Perspectives of Marine Container Ports: A Case Study for Turkey's Container Ports and Terminals. Journal of Yaşar University, 14, 33-47.

[126]Notteboom, T., Ducruet, C., \& De Langen, P. (2016). Ports in proximity: Competition and coordination among adjacent seaports. London: Routledge.

[127]Darbra, R. M., \& Casal, J. (2004). Historical analysis of accidents in seaports. Safety Science, 42(2), 85-98.

[128]Yip, T. L. (2008). Port traffic risks-A study of accidents in Hong Kong waters. Transportation Research Part E: Logistics and Transportation Review, 44(5), 921-931.

[129]Mollaoğlu, M., Bucak, U., \& Demirel, H. (2019). A quantitative analysis of the factors that may cause occupational accidents at ports. Journal of ETA Maritime Science, 7(4), 294-303.

[130]Bichou, K. (2013). Port operations, planning and logistics. New York: Informa Law from Rutledge. 